This is an Accepted Manuscript of an article published by Taylor \& Francis Acta Odontologica Scandinavica. 2017.75(5):372-375. Published online 22 Apr 2017. Available online: http://dx.doi.org/10.1080/00016357.2017.1317830.

\title{
Duration of orthognathic-surgical treatment
}

Jaakko Paunonen, ${ }^{a}$ M ika Helminen, ${ }^{b}$ Timo Peltomäkia,c

a DDS, M axillofacial surgeon, Department of Ear and Oral Diseases, Tampere University Hospital, Tampere, Finland

b M.Sc., Biostatistician, Science Center, Pirkanmaa Hospital District and School of Health Sciences, University of Tampere, Finland

a,c Professor, Field of Dentistry, Faculty of M edicine and Life Sciences, University of Tampere, Finland

Address correspondence to: Timo Peltomäki, Department of Ear and Oral Diseases, Tampere University Hospital, and Field of Dentistry, Faculty of M edicine and Life Sciences, University of Tampere, Biokatu 6 B, 33520 Tampere, Finland; e-mail: timo.peltomaki@pshp.fi, telephone +358 405668056. 


\section{Duration of orthognathic-surgical treatment}

\section{Introduction}

Orthognathic-surgical treatment is routine practice worldwide to correct dentoskeletal malocclusions in adults. Since orthognathic-surgical treatment is elective, not obligatory or lifesaving, the patient's own opinion regarding whether or not to commence treatment is of utmost importance. During the decision-making process, a patient should clearly understand what the treatment entails, as well as the goals, achievable outcome and duration of treatment. According to Cunningham and Shute [1] patient satisfaction is based on four main factors: a technically good result, internal patient-related factors, interaction and communication, and external factors connected to the patient and treatment team. Of these, "interaction and communication" at all stages of treatment seems to play a key role in achieving post-treatment satisfaction. A technically good result is not a guarantee of satisfaction, if pre-treatment information and communication of the treatment course and goals were deficient.[1] Since most orthognathic-surgical patients are adults, it is important to give as precise an estimation of treatment duration as possible. In addition to changes in facial appearance, long treatment duration may affect patients' post-treatment satisfaction.[2] It is also noteworthy that many adult patients consider fixed orthodontic therapy the most disruptive part of the treatment.[3] Even oral health-related quality of life has been found to worsen during the initial stages of the fixed appliance treatment.[4]

Factors that have been suggested to affect orthognathic-surgical treatment duration include age, gender, co-operation, severity/type of malocclusion, treatment method (tooth extractions, type of surgery), and practice setting (university/hospital, private, experience of orthodontist).[5-14]

The aim of this study was to determine the duration of orthognathic-surgical treatment conducted with conventional pre- and post-surgical orthodontic treatment phases.

\section{Material and methods}

The study material was comprised of the files of 185 consecutive patients with full set of good quality documents and who had undergone orthognathic treatment at the Oral and Maxillofacial Unit of Tampere University Hospital, Tampere, Finland, in 2007-2014. Patients with cleft lip and palate or craniofacial anomalies and patient whose orthodontic treatment was made outside the 
Hospital were excluded. Ethical approval was obtained from the Ethics Review Committee of the joint M unicipal Authority of the Pirkanmaa Hospital District. The files were reviewed and the following data was obtained: gender, ICD-10 diagnosis, type of malocclusion, age at the initiation of treatment (usually placement of separating rings for banding), duration of pre-surgical treatment (until operation), type of operation (bilateral sagittal split osteotomy, LeFort 1 osteotomy or bimaxillary surgery), and duration of post-surgical treatment (from date of operation until date of fixed orthodontic appliance removal and delivery/placement of retainers). In addition, information about and reason (decompensation or crowding) for orthodontic tooth extractions (excluding $3^{\text {rd }}$ molars) were retrieved. Pre-treatment digital lateral head radiograms were used to study the severity of the skeletal malocclusion. The cephalograms were examined by one investigator (JP) using Planmeca Romexis software (Planmeca, Finland). The following skeletal and dental measurements were included: SNA, SNB and ANB angles for sagittal maxillary and mandibular position, and their relationship; vertical relationship between palatal and mandibular planes (NL-M L); anterior facial height ( $\mathrm{Na}-\mathrm{Me}$ ); length of the maxilla (Co-A) and mandible (Co-Gn); and inclination of upper and lower incisors (UI-NL and LI-ML). All cephalograms were measured twice, and the mean was used in the statistical analyses.

Four experienced oral and maxillofacial surgeons had operated on the patients. Orthodontic treatment had been provided by three experienced orthodontists, or, in the case of postgraduate students, had been closely supervised by experienced orthodontists. Straight-wire orthodontic technique was used with Roth's bracket prescription, and the patients were seen every four-six weeks. All patients were treated by orthodontics to achieve the best possible post-operative occlusion, and were evaluated jointly by the treating orthodontist and oral and maxillofacial surgeon about three months before the operation to ensure best possible post-operative occlusal stability. In all cases surgical splint was used at the operation to obtain the planned occlusion.

Fisher's Exact Test was used in the comparison of categorical variables in cross tabulation. Differences in continuous variables between groups were tested with the Mann-Whitney U test. In addition, treatment duration variables (pre-, post- and total) were used as dependent variables in separate linear regression models, with type of surgery, orthodontic extractions, age at the initiation of treatment, gender and cephalometric results as independent variables (IBM SPSS Statistics, V22.0, Armonk, NY). 


\section{Results}

Distribution of patients by gender showed that $61 \%$ were female. The mean age of the sample at the initiation of treatment was 32.1 years (range 17-70 years). The most common skeletal diagnosis was mandibular retrognathia, in $64 \%$ of cases (119/185, ICD-10: K07.13); $20.5 \%$ (38/185) had mandibular prognathia (ICD: K07.11). Type of operation was distributed as follows: 103 patients had bilateral sagittal split osteotomy (62\% female, mean age 36.5 years); 37 had LeFort 1 osteotomy ( $73 \%$ female, mean age 25.5 years); and 45 had bimaxillary surgery ( $51 \%$ female, mean age 34.3 years).

Total treatment duration (median, Q1-Q3) from placement of separating rings for banding until fixed orthodontic appliances were removed and retention period started was 31.1 months (24.541.8 months), of which pre-surgical orthodontics took 24.4 months (18.0-32.6 months) and postsurgical 6.4 months (4.6-9.2 months). Treatment duration (median) was nearly the same for each type of surgery: bilateral sagittal split osteotomy was 32.1 months, LeFort 1 osteotomy 30.1 months, and bimaxillary osteotomy 29.7 months (Figure 1).

Extractions were performed in 35 patients (19\%) as part of orthodontic treatment. If the orthodontic treatment included tooth extraction, the duration of pre-surgical treatment was on average 10 months longer, which is a statistically highly significant difference $(p<0.001$, linear regression; Figure 2). The reasons for extraction were crowding (20 patients) and decompensation (15 patients), and no statistically significant difference in treatment duration was found between these two groups. Patients with extractions were distributed according to type of surgery, as follows: $17.5 \%$ had BSSO, $13.5 \%$ had LeFort 1 , and $26.7 \%$ had bimaxillary surgery - a not statistically significant difference. The most commonly extracted teeth were first or second lower bicuspids in BSSO cases, or solely upper first bicuspids in LeFort I cases, whereas the extracted teeth varied in bimaxillary cases. Duration of post-surgical orthodontic treatment did not vary in a statistically significant way between extraction and non-extraction individuals. No statistically significant relation was found between treatment time and age of patients or initial severity of malocclusion. Figure 3 shows scatterplot between pre-surgical treatment time (cases with and without extractions separately) and ANB angle; no statistically significant association was found. 


\section{Discussion}

The present patient population is a typical Finnish orthognathic-surgical patient pool in terms of gender distribution: 61\% female [15-18] The present sample differs, however, from a typical sample in terms of age distribution: mean age (32.1 years) at the initiation of treatment was greater than in most other reports [19], and the oldest patient was 70 years of age. Interestingly, patients with LeFort I maxillary advancement were younger on average than patients with other types of surgery. This difference may be related to aesthetically motivated requests for earlier treatment on the part of younger individuals, especially in light of the large proportion of female patients (73\%). In our sample, mandibular retrognathia was the most common diagnosis and mandibular lengthening with BSSO the most common operation.

Since reliable information and communication before treatment are important for post-treatment satisfaction, the knowledge shared with patients should be based on scientific evidence. One example is treatment duration, which is often estimated too optimistically [11] and is a key issue in the decision-making process for opting in or out of treatment.[20] In the present study, the median pre-surgical treatment time was 24.4 months, which compares fairly well with that of previous studies; however, our treatment time was near the upper limit.[5,7-8,10,12,14] In the present study, pre-surgical treatment time was prolonged maximally by $1-2$ months until access to surgery was possible. Post-surgical orthodontic treatment was initiated usually four weeks after the operation and lasted an average of about six months (median), which is shorter than the ninemonth critical period for patients' acceptance post-surgery.[21]

In the present study, tooth extraction as part of the orthodontic treatment was the only factor to prolong treatment duration. According to our findings, pre-surgical time was on average 10 months longer with extractions, which is in line with an earlier report [9], but still significantly longer than the five-month prolonging reported by Dowling et al.[13] One to two month waiting period until access to surgery means that active per-surgical orthodontic treatment lasted 8-9 months longer with extractions. Duration of post-surgical orthodontic treatment did not vary between extraction and non-extraction individuals which makes sense since extraction spaces are mostly closed during the pre-surgical orthodontic phase. The reason for tooth extraction was not found to make a difference in treatment duration, and, in any event, the reason is frequently a combination of decompensation and crowding. The extraction pattern in our findings was 
consistent with the recommended extraction arrangement in orthognathic patients.[22,23] In mandibular advancement cases with BSSO, first or second lower bicuspids were extracted, and in LeFort I advancement cases, upper first bicuspids.

It is commonly thought that orthodontic treatment time is related to an individual's age, and that older patients have a longer duration. This was not found in the present study despite the patients' wide age range (17-70 years), which allowed a study of the association between age and treatment duration. Lack of co-operation (forgotten or cancelled appointments) could also prolong duration, but is seldom an issue in adult patients.

In some studies, severity of pre-treatment skeletal discrepancy has been found to be associated with treatment duration $[8,11]$, but this was not the case in the present study. It is reasonable to expect that initial severity has an impact on treatment duration, when orthodontics only are used for the correction. $[24,25]$ In orthognathic treatment, the severity of the initial condition does not seem to be an influential variable on treatment duration, since, for example, it takes a surgeon the same time to advance the mandible 6 or $12 \mathrm{~mm}$. The experience of different surgeons would only have an impact on the duration of the operation, not on the treatment as a whole. In the present case, all of the surgeons were experienced, with several years of clinical practice. Based on a recent systematic review, the average treatment duration with fixed orthodontic appliances was 20 months [20], which is fairly consistent with the present pre-surgical treatment time.

The practice setting and the experience of an orthodontist have been found to impact treatment duration; for example, treatment at university clinics with experienced orthodontists leads to shorter duration. $[5,7,8,12,13,14]$ In the present study, this issue could not be properly studied since all patients were treated at the university clinic by experienced orthodontists.

Over the past decade, two approaches have been introduced with the aim of shortening treatment times: surgery first, as well as various surgical and non-surgical means to accelerate orthodontic tooth movement. In a recent systematic review [26], treatment duration in the surgery first approach was found to be an average of 14.2 months, which is significantly less than in the conventional approach reported previously as well as here, or when distraction osteogenesis has been used as the operation technique for mandibular advancement.[27] Despite promising means of accelerating orthodontic tooth movement, there is still limited - if any - 
scientific evidence to support the use of these techniques in reducing treatment time.[28,29] In the present study, conventional pre- and post-surgical orthodontic treatment with straight-wire technique was applied in all cases.

To conclude, the present findings can serve as reliable pre-treatment information regarding the expected duration of orthognathic treatment, since duration is one of the most important factors taken into consideration by adult patients deciding whether or not to commence treatment. Tooth extractions (excluding $3^{\text {rd }}$ molars) included in pre-surgical orthodontic treatment prolong treatment time by an average of 8-9 months.

\section{Disclosure statement}

The authors report no conflicts of interest. 


\section{References}

1. Cunningham SJ, Shute J. Orthognathic treatment: see how they feel? J Orthod. 2009; 36: 61-66.

2. Khattak ZG, Benington PC, Khambay BS, et al. As assessment of the quality of care provided to orthognathic surgery patients through a multidiscliplinary clinic. J Craniomaxillofac Surg. 2012; 40:243-247.

3. Nurminen L, Pietilä T, Vinkka-Puhakka H. M otivation for and satisfaction with orthodontic-surgical treatment: a retrospective study of 28 patients. Eur J Orthod. 1999;21:79-87.

4. Johal A, Joury E. What factors predict the uptake of orthodontic treatment among adults? Am J Orthod Dentofacial Orthop. 2015;147:704-710.

5. Luther F, M orris DO, Hart C. Orthodontic preparation for orthognathic surgery: how long does it take and why? A retrospective study. Br J Oral M axillofac Surg. 2003;41:401-406.

6. Luther F, M orris DO, Karnezi K. Orthodontic treatment following orthognathic surgery: how long does it take and why? A retrospective study. J Oral Maxillofac Surg. 2007;65:1969-1976.

7. Mavreas $D$, Athanasiou $A E$, Factors affecting the duration of orthodontic treatment: a systematic review. Eur J Orthod. 2008;30:386-395.

8. Jeremiah HG, Cousley RR, Newton T, et al. Treatment time and occlusal outcome of orthognathic therapy in the East of England region. J Orthod. 2012;39:206-211.

9. Häll B, Jämsä T, Soukka T, Peltomäki T. Duration of surgical- orthodontic treatment. Acta Odontol Scand. 2008;66:274-277.

10. Diaz PM, Garcia RG, Gias LN, et al. Time used for orthodontic surgical treatment of dentofacial deformities in white patients. J Oral M axillofac Surg. 2010;68:88-92.

11. O'Brien K, Wright J, Conboy F, et al. Prospective, multi-center study of the effectiveness of orthodontic/orthognathic surgery care in the United Kingdom. Am J Orthod Dentofacial Orthop. 2009;135:709-714.

12. Proffit WR, Miguel JA. The duration and sequencing of surgical-orthodontic treatment. Int J Adult Orthodon Orthognath Surg. 1995;10:35-42.

13. Dowling PA, Espeland L, Krogstad O, et al. Duration of orthodontic treatment involving othognathic surgery. Int J Adult Orthodon Orthognath Surg. 1999;14:146-152.

14. Slavnic S, Marcusson A. Duration of orthodontic treatment in conjunction with orthognathic surgery. Swed Dent J. 2010;34:159-166.

15. Forssell H, Finne K, Forssell K, et al. Expectations and perceptions regrading treatment: a prospective study of patients undergoing orthognathic surgery. Int J Adult Orthodon Orthognath Surg. 1998;13:107-113

16. Pahkala RH, Kellokoski JK. Surgical-orthodontic treatment and patients` functional and psychosocial well-being. Am J Orthod Dentofacial Orthop. 2007;132:158-164. 
17. Silvola AS, Varimo $M$, Tolvanen $M$, et al. Dental esthetics and quality of life in adults wiht severe malocclusion before and after treatment. Angle Orthod. 2014;84:594-599.

18. Svedstöm-Oristo AL, Ekholm H, Tolvanen M, et al. Self-reported temporomandibular disorder symptoms and severity of malocclusion in prospective orthognathic-surgical patients. Acta Odontol Scand.

2016;74:466-470.

19. Veldhuis EC, Veldhuis AH, Bramer WM, et al. The effect of orthognathic surgery on temporomandibular joint and oral function: a systematic review. Int J Oral Maxillofac Surg. 2017 Feb 4, (17)30021-8.

20. Tsichlaki A, Chin SY, Pandis N, et al. How long does treatment with orthodontic appliances last? A systematic review. Am J Orthod Dentofacial Orthop. 2016;149:308-318.

21. Kiyak HA, Hohl T, West RA, et al. Psychologic changes in orthognathic surgery patients: a 24-month follow up. J Oral M axillofac Surg. 1984; 42:506-512.

22. Proffit WR, White RP, Sarver DM. Contemporary Treatment of Dentofacial Deformity. St. Louis, M osby. 2003

23. Proffit WR, White RP Jr. Combined surgical-orthodontic treatment: how did it evolve and what are the best practices now? Am J Orthod Dentofacial Orthop. 2015;147:S205-15.

24. Vig PS, Weintraub JA, Brown C, et al. The duration of orthodontic treatment with and without extractions: a pilot study of five selected practices. Am J Orthod Dentofacial Orthop. 1990;97:45-51.

25. Skidmore KJ, Brook KJ, Thomson WJ, et al. Factors influencing treatment time in orthodontic patients. Am J Orthod Dentofacial Orthop. 2006;129:230-238.

26. Peirò-Guijarro M A, Guijarro-M artìnez R, Hernàndez-Alfaro F. Surgery first in orthognathic surgery: $A$ systematic review of the literature. Am J Orthod Dentofacial Orthop. 2016; 149:448-462.

27. Breuning KH, van Strijen PJ, Prahl-Andersen B, et al. Duration of orthodontic treatment and mandibular lengthening by means of distraction or bilateral sagittal split osteotomy in patients with Angle Class II malocclusions. Am J Orthod Dentofacial Orthop. 2005;127:25-29.

28. Fleming PS, Fedorowicz Z, Johal A, et al. Surgical adjunctive procedures for accelerating orthodontic treatment. Cochrane Database Syst Rev. 2015;30:CD010572.

29. Woodhouse NR, DiBiase AT, Johson N, et al. Supplemental vibrational force during orthodontic alignement: a randomized trial. J Dent Res. 2015;94:682-689. 


\section{Figure texts}

Figure 1. Box-plots showing pre- and post-operative treatment time in months for each surgery type

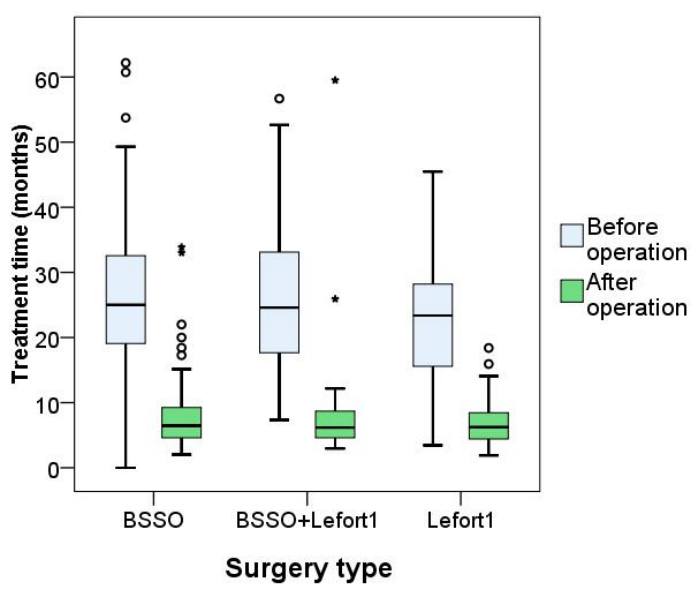

Figure 2. Box-plots showing the relationship between tooth extractions and pre-surgical treatment time in months.

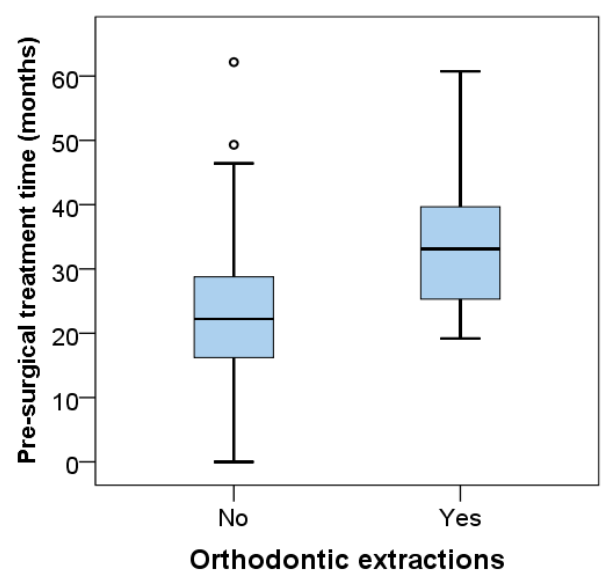

Figure 3. Scatterplot between pre-surgical treatment time with and without tooth extractions and ANB angle.

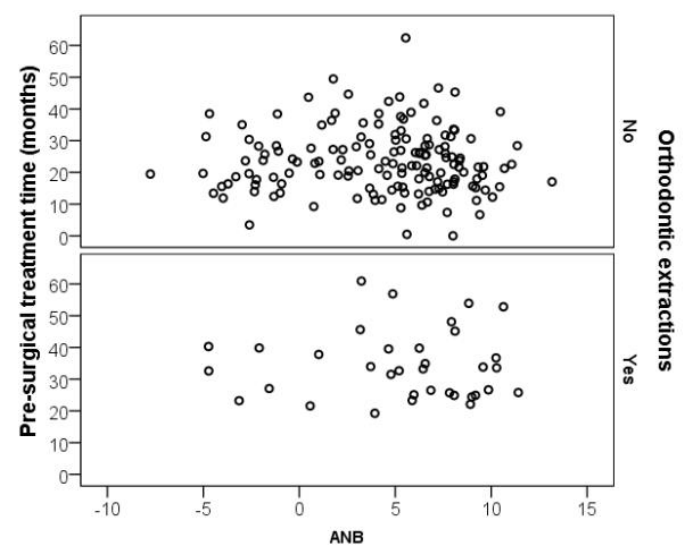

